\title{
QUALIDADE DA INFORMAÇÃO CONTÁBIL: ANÁLISE BIBLIOMÉTRICA DAS PESQUISAS EM COOPERATIVAS
}

\section{ACCOUNTING INFORMATION QUALITY: BIBLIOMETRIC ANALYSIS OF RESEARCH IN COOPERATIVES}

\begin{abstract}
0 artigo foi aprovado e apresentado no XX USP International Conference in Accounting, realizado de 29 a 31 de julho de 2020, em São Paulo (SP), com participação online devido à pandemia da COVID-19.
\end{abstract}

\section{RESUMO}

Esta pesquisa teve como objetivo verificar o cenário das pesquisas sobre qualidade da informação contábil em cooperativas. Com direcionamento para a abordagem quanti-quali, utilizou-se informações sobre produção nacional e internacional no período de 2010 a 2020, foram pesquisadas as bases Spell e Scholar Google, para estudos nacionais, e Web of Science, para os internacionais. O estudo se justificou devido à importância do cooperativismo na economia mundial e nacional. Realizou-se um levantamento dos periódicos com maiores publicações, os autores com maior produtividade sobre o tema, a frequência de palavras e, quais das métricas de mensuração da qualidade da informação foram mais utilizadas. Como resultado identificou-se que alguns autores se destacam, e o vínculo dos autores com maior número de publicações está ligado à Universidade Federal de Minas Gerais. Os resultados apontaram que o gerenciamento de resultados foi a principal métrica de qualidade de informação, e cooperativas de crédito foi a população mais explorada nas pesquisas. Não há predominância de modelos utilizados, porém foi possível verificar que a proxy qualidade da informação é utilizada como consequente (variável dependente), tal resultado poderia se justificar devido à disponibilidade de dados por parte das cooperativas de créditos e por elas se enquadrarem no setor de instituições financeiras, além de serem mais suscetíveis ao gerenciamento de resultados, como no caso dos bancos. $\mathrm{O}$ artigo contribuiu com um panorama sobre qualidade da informação contábil nas cooperativas, a nível nacional e internacional.

Palavras-chave: Bibliometria; Qualidade da Informação Contábil; Cooperativas.

\section{ABSTRACT}

This research aimed to verify the scenario of research on the quality of accounting information in cooperatives. With a focus on the quanti-quali approach, information on national and international production was used in the period from 2010 to 2020, the Spell and Scholar Google databases were researched for national studies, and Web of Science for international ones. The study was justified due to the importance of corporativism in the world and national economy. A survey was conducted of the journals with the largest publications, the authors with the highest productivity on the topic, the frequency of words and which of the metrics used to measure the quality of information were most used. As a result, it was identified that some authors stand out, and the link of the authors with the largest number of publications is linked to the Federal University of Minas Gerais. The results showed that earnings management was the main metric for information quality, and credit unions were the most explored population in the surveys. There is no predominance of models used, but it was possible to verify that the information quality proxy is used as a consequent (dependent variable), such a result could be justified due to the availability of data by the credit unions and because they fit into the credit sector. financial institutions, in addition to being more susceptible to earnings management, as in the case of banks. The article contributed to an overview of the quality of accounting information in cooperatives, both nationally and internationally.

Keywords: Bibliometry; Quality of Accounting Information; Cooperatives

\section{Guilherme Domiciano Ferreira}

Mestrando no Programa de Pós-Graduação da Universidade Federal de Goiás, possui graduação em Ciências Contábeis pela mesma universidade, pós-graduado em Auditoria Digital e Direito Tributário. Sócio da empresa Inov Serviços e Soluções em Contabilidade. E-mail: guilherme.contabilidade@outlook.com

\section{Carlos Henrique Silva do Carmo}

Doutor em Controladoria e Contabilidade pela Faculdade de Economia, Administração e Contabilidade da Universidade de São Paulo - FEA/USP (2014). Mestre em Engenharia de Produção pela Universidade Federal de Santa Catarina - UFSC (2003) e Graduado em Ciências Contábeis pela Pontifícia Universidade Católica de Goiás - PUC-GO (1999). É professor da Faculdade de Administração, Ciências Contábeis e Ciências Econômicas da Universidade Federal de Goiás - FACE/UFG (Desde 2006), onde exerceu a coordenação do Mestrado em Ciências Contábeis (2016-2020). É coordenador de Pesquisa e participante do Centro de Estudos, Pesquisa e Extensão do Programa de Pós-graduação em Ciências Contábeis da UFG CEPECONF. E-mail: chscarmo@ufg.br

\section{Ercilio Zanolla}

Doutor em Ciências Contábeis pelo Programa Multiinstitucional e Inter-regional de Pós-graduação em Ciências Contábeis UnB/UFPB/UFRN (2014). Mestre em Ciências Contábeis pela Universidade do Estado do Rio de Janeiro (2003). Possui graduação em Ciências Contábeis pela Universidade Federal de Mato Grosso do Sul (1993). Professor do Curso de Ciências Contábeis da Faculdade de Administração, Ciências Contábeis e Ciências Econômicas da Universidade Federal de Goiás - FACE/ UFG. Professor do Programa de Pós-graduação em Ciências Contábeis da UFG - PPGCONT/UFG. Elaborou o Projeto de criação do Curso de Graduação em Ciências Contábeis da UFG (2006) o qual coordenou até 2010. E-mail: zanolla@ufg.br

\section{Paulo Junio Pereira de Moura}

Mestre em Ciências Contábeis pela Universidade Federal de Goiás. Especialização em Docência do Ensino Superior pela Faculdade Brasileira de Educação e Cultura (2016). Graduação em Ciências Contábeis pela Universidade Federal de Goiás (2015). Professor do Centro Universitário Alves Faria (UNIALFA) e na Escola Superior Associada de Goiânia (ESUP). Sócio na empresa: Lupa Assessoria e Gestão de Negócios Ltda. E-mail paulo-moura@outlook.com

\section{Denise Fernandes Nascimento}

Mestrado em Ciências Contábeis pela Universidade Federal de Goiás, Brasil (2018). Mestrado em Administração pelo Centro Universitário Alves Farias - UNIALFA, Brasil (2015) Especialização em Gestão Pública pela Faculdade Brasileira de Educação e Cultura - FABEC (2011). Graduação em Ciências Contábeis pelo Centro Universitário de Goiás Uni-ANHANGUERA (1999). Professora no Curso de Ciências Contábeis do Centro Universitário Alves Faria (UNIALFA) Coordenadora e Professora da Escola Superior Associada de Goiânia ESUP - FGV (conveniada). Analista em Organização e Finanças - Contadora, da Prefeitura de Goiânia, exercendo a atividade de Auditoria e Controle Interno, na Controladoria Geral do Município - CGM. E-mail: denisefn29@gmail.com 


\section{INTRODUÇÃO}

Conforme dados da Organização das Cooperativas Brasileiras (OCB) (2020) existem cerca de 3 milhões de cooperativas no mundo, que são responsáveis por gerar 280 milhões de empregos e 1,2 bilhão de cooperados.

De acordo com a OCB (2019) para se compreender a influência das cooperativas, a cada sete pessoas no mundo, uma é associada à cooperativa. Segundo dados da OCB, caso se juntassem as 300 maiores cooperativas do mundo em um único país, seriam a $9^{\mathrm{a}}$ economia mundial. Atualmente, no Brasil existem cerca de 6.828 cooperativas e juntas representam um ativo total de $\mathrm{R} \$ 351,4$ bilhões e cerca de 14,6 milhões de cooperados (OCB, 2019).

Com o passar dos anos, o sistema de cooperativas foi se aprimorando em sua complexidade estrutural. Em razão do aumento da complexidade na gestão das cooperativas, torna-se importante a utilização de mecanismos para mitigar os problemas de agência (Staatz, 1987).

Como a exigência passou a ser maior devido ao crescimento do modelo de cooperativismo, surgiu a necessidade de adesão de profissionais especializados para auxiliar na gestão das cooperativas, como administradores das entidades (Zylbersztajn, 1994).

O dever de controle da cooperativa é dos seus cooperados, isso porque a cooperativa tem a função de servir às necessidades dos cooperados (Dunn, 1988). Porém, há dificuldades na forma de manutenção da democracia entre os membros, ocorrendo problemas de agência e assimetria informacional entre conselho e membros (Osterberg \& Nilsson, 2009).

O conselho de administração deve buscar sua legitimidade para evitar conflitos com seus membros, e uma das formas de garantir sua legitimação é por meio da prestação de contas (accountability) para se obter informações com maior transparência e qualidade (Silva, Sousa \& Leite, 2010).

De acordo com o conselho de administração, é importante que a cooperativa tenha resultados positivos para demonstrar a eficiência na gestão (Bressan, Bressan \& Junior, 2015). Por outro lado, um resultado muito positivo pode demonstrar uma certa "exploração" dos cooperados (principal) que, na maioria das vezes, são os fornecedores e os consumidores da própria cooperativa.

Nesse contexto, a contabilidade introduz uma forma de controle dos contratos entre os agentes envolvidos (Lopes \& Martins, 2005). Para a diminuição da assimetria informacional entre cooperados e gestores na tomada de decisão e na gestão dos contratos, é necessário que a informação contábil seja de qualidade.

Lopes (2009) usou uma proxy para medir a qualidade da informação (informativeness) de acordo com a abordagem de portifólio. Nessa abordagem, são utilizados os seguintes métodos na verificação da qualidade da informação: Value relevance (relevância), Conservatism (conservadorismo), Timeliness (tempestividade) e Earnings Management (gerenciamento de resultados), sendo a relevância, a tempestividade e o conservadorismo responsáveis por capturar a realidade econômica nos relatórios contábeis (Lopes, 2009).

As cooperativas são entidades econômicas que possuem a finalidade de suprir as falhas do mercado (Souza Junior, Costa, Richetti \& Asta, 2018). As cooperativas agrícolas têm uma importante função na coordenação de processos agroalimentares dentro de suas regiões (Zylbersztajn, 1994). Já as cooperativas de crédito têm um papel relevante no desenvolvimento social e local (Santos, Bressan, Braga, \& Guerra, 2018).

A composição de uma cooperativa é caracterizada pela figura do cooperado que, na maioria das vezes, comercializa sua produção com a cooperativa e busca o retorno do investimento realizado na entidade por meio das sobras, que seriam o "lucro". Nesse cenário, o cooperado é proprietário e fornecedor da própria cooperativa.

Diante deste contexto, e devido às grandes possibilidades de pesquisas relacionadas a cooperativas e considerando a relevância da temática, o presente estudo visa responder ao seguinte problema: Qual o cenário das pesquisas sobre qualidade da informação contábil em cooperativas?

Neste sentido o objetivo geral do estudo é verificar o cenário das pesquisas sobre qualidade da informação contábil em cooperativas.

Muitos estudos têm abordado aspectos ligados ao comportamento dos cooperados (Gomes, Lima, Araújo e Asta, 2019). Outras pesquisas abordam o tamanho da cooperativa, idade dos cooperados, níves de preços, variáveis sociopsicológicas, como por exemplo: a falta de coesão e compromisso social.

Gomes et al. (2019) buscaram verificar quais eram os temas de pesquisas com cooperativas no exterior, e identificaram quatro assuntos principais: conflito de interesses, considerações metodológicas, preocupações geopolíticas e modelos matemáticos.

A identificação de pesquisas com cooperativas e das temáticas envolvidas, permitem uma ampla visão sobre o assunto, e consequentemente a consolidação dos principais achados dos estudos. A análise dos assuntos relacionados aos conflitos de interesses, no meio pesquisado, permite conhecer mais sobre as fontes de informações, sobre as cooperativas e o mercado em que estão inseridas.

Além disso, os estudos bibliométricos podem auxiliar na compreensão e análise do campo pesquisado e das obras citadas com o intuito de se conhecer autores e os temas qua abordam (Da Silva Vicente, Rafael, Serra, \& Almeida, 2020). 


\section{REFERENCIAL TEÓRICO}

O referencial teórico abordam-se os seguintes tópicos: o cooperativismo e a contabilidade e qualidade da informação contábil, assuntos relevantes para melhor compreensão do estudo.

\section{O Cooperativismo e a Contabilidade}

Uma cooperativa é definida como uma organização das quais as sobras são exclusivas dos agentes que abastecem com patrocínio sob a lógica de contratos da entidade (cooperados) e o conselho administrativo seria eleito por esses cooperados (Vitaliano, 1983). No cenário internacional, a maioria das cooperativas são administradas por especialistas sem vínculo cooperativo com a entidade.

Em meados de 1960, especulou-se que a modernização da agricultura seria o fim das cooperativas agrícolas, porém alguns autores sugeriam que as cooperativas iriam evoluir tornando-se uma agência de integração dos agricultores (Cook, 1995).

Até o ano de 1993, aproximadamente 30\% das vendas da produção agrícola norte-americana seriam geradas pelas cooperativas (Cook, 1995). A perspectiva é de que as cooperativas corrijam as falhas de mercado com uma reestruturação de estratégias.

Muitas cooperativas têm enfrentado desafios financeiros e organizacionais, causados internamente por heterogeneidade entre os cooperados, problemas de direitos de propriedade e por queda no comprometimento dos membros (Fulton e Giannakas, 2001).

Nas cooperativas os conselhos de administração devem prestar contas para os cooperados. A prestação de contas é a finalização de todo o ciclo operacional, na qual a administração informa, por meio das demonstrações contábeis, o cumprimento das metas estabelecidas (Ribeiro Filho, 1993).

Conforme a Lei 5.764/71 a prestação de contas é importante pois as sobras apuradas são destinadas aos cooperados e as perdas devem ser rateadas entre eles. Por isso, é importante que as informações demonstradas pela contabilidade satisfaçam os usuários para que eles possam tomar decisões sobre os objetivos da cooperativa.

Uma outra particularidade do cooperativismo, que está ligada a seu processo contábil, é o reconhecimento do ato cooperado e não cooperado. As operações com cooperados e não cooperados devem ser contabilizadas separadamente, pois esse dado é uma importante informação para o cálculo dos tributos, para tomada de decisão e prestações de contas (Domingues, 2003).

Outro fato, diz respeito a modernização, que no modelo cooperativista, pós industrialização, tem gerado discussões sobre a proximidade com o modelo de gerenciamento das grandes corporações, com necessidade de informações e aumento dos ganhos (Cook, Chaddad \& Iliopoulus, 2004).

Para Santos et al. (2018) o cooperativismo é um instrumento que busca agregar bem-estar social e desenvolvimento econômico. A forma de se atender esses dois requisitos é por meio da união de pessoas com o mesmo objetivo.

A utilização da contabilidade nas cooperativas tem como motivação a obtenção de informações, relação de custo-benefício e o interesse da alta administração. Já a falta de tecnologia e experiência da equipe interna da cooperativa são fatores que restringem a utilização de dados contábeis nas entidades (Imlau, 2015).

Tarifa e Almeida (2019) buscaram evidenciar a tipificação cultural na contabilidade gerencial das cooperativas agropecuárias do Paraná. Os resultados da pesquisa, apontam que a cultura denominada como clã ou grupal estaria presente na maior parte da amostra estudada. Essa cultura estaria ligada a organizações com cunho familiar, nas quais a cooperativa seria uma "extensão da família", considerando a lealdade entre os membros e o tradicionalismo.

Vitaliano (1983) constatou que de 1940 a 1960 pouco progresso havia sido gerado no desenvolvimento de uma teoria cooperativa, porém, com o desenvolvimento da teoria neoclássica e com a criação da nova economia institucional, o autor aponta que novos conceitos foram integrados à teoria cooperativa.

\subsection{Qualidade da informação contábil}

A informação contábil de qualidade é a que demonstra o desempenho financeiro de uma entidade e que é relevante para a tomada de decisão (Dechow, Ge \& Schrand, 2010).

Inseridos nesta definição, estão três fatores. O primeiro é de que a qualidade depende da relevância da decisão. O segundo, qualidade depende se é informado o desempenho financeiro da empresa, pois existem aspectos não observáveis e por fim, a qualidade será um conjunto entre a relevância do desempenho financeiro após a decisão e a capacidade da contabilidade em medir o desempenho.

Percebe-se que a qualidade da informação tem sido objeto de estudos de vários pesquisadores sob a perspectiva da tempestividade da informação. A tempestividade da divulgação da informação estaria também ligada ao conservadorismo (Kothari, Ramanna \& Skinner, 2009).

Ressalta-se que na literatura não há consenso sobre como medir a qualidade da informação, porém, alguns pesquisadores encontraram as seguintes métricas para mensurar a qualidade, são elas: nível de conservadorismo, relevância da informação, tempestitividade, gerenciamento de resultados (Wang, 2006; Lopes, 2009). 
A relevância da informação contábil teve seu pontapé inicial com as pesquisas de Ball e Brown (1968) e Beaver (1968), nas quais foram medidas as reações do mercado à divulgação dos ganhos. A relevância é a métrica da qualidade da informação que analisa a relação entre números contábeis e o valor de mercado (Barth, Beaver \& Landsman, 2008).

Conforme Ahmed (1994) o conservadorismo acontece quando os gestores deixam de reconhecer uma oportunidade de crescimento nas demonstrações. Para Van Breda e Hendriksen (1997) o conservadorismo está presente na orientação dos contadores em reconhecerem um ativo pelo menor valor e um passivo pelo maior. Os autores apontam que essa medida reduziria o otimismo dos investidores, porém seria uma distorção dos dados contábeis.

Lopes (2009) relacionou a tempestividade a padrões de governança, Almeida (2010) aos ambientes competitivos e Silva (2013) relacionou a tempestividade à adoção das normas internacionais (Silva, 2013).

De acordo com Paulo (2007) o gerenciamento de resultados pode acontecer de três formas: a primeira pelas escolhas contábeis (geralmente, accruals). A segunda em atividades operacionais e a terceira na manipulação classificatória nas demonstrações contábeis. O autor define, então, que o gerenciamento é a manipulação dos dados, por meio das métricas apontadas, na qual o gestor pode ser motivado por diversas razões a gerenciar resultados, seja por pressão, seja por reputação, além do próprio incentivo financeiro.

Para Almeida (2010) existe uma relação direta entre a competitividade e a qualidade da informação contábil. O autor constatou que quanto maior a competitividade do ambiente em que a empresa está inserida, menor a relevância da informação e o aumento da tempestividade.

A relevância estaria ligada de forma positiva ao nível de governança corporativa (Almeida, 2010). O autor constatou que o conservadorismo cresce na medida em que se aumenta a competitividade do ambiente, isso implica nos insiders que acabam optando por reconhecerem más notícias futuras antecipadamente. Já em relação ao gerenciamento de resultados, o autor revela o contrário das métricas anteriores, o gerenciamento de resultados, tem uma relação inversa com a competitividade, esperando-se que em ambientes mais competitivos tenha menos espaços para a manipulação das informações.

Assim, pelos estudos apresentados, percebe-se a necessidade de se identificar o cenário das pesquisas de qualidade da informação em cooperativas, uma vez que se trata de importantes e lucrativas organizações. Embora as cooperativas não possuam ações negociadas em bolsas de valores, os investidores de capital social podem perceber de forma positiva a persistência das sobras líquidas do exercícios, ou outros investimentos oferecidos pelas cooperativas (Diniz, 2020).

\section{PROCEDIMENTOS METODOLÓGICOS}

O objetivo deste artigo foi analisar a produção científica sobre qualidade da informação contábil em Cooperativas no contexto nacional e internacional, e, para tanto utilizou-se do estudo bibliométrico, que tem como função quantificar, descrever e fornecer prognósticos em relação ao processo de comunicação escrita (Beuren \& Sousa, 2007).

A abordagem, adota o enfoque quali-quanti, como o utilizado por Blonkoski, Antonelli e Bortoluzzi (2016), tendo a parte quantitativa sido realizada pela análise bibliométrica, para quantificar a produção científica a respeito da qualidade da informação contábil por meio dos artigos selecionados, e, a parte qualitativa ocorreu via análise do conteúdo dos artigos, buscando conhecer as bases teóricas e contextos que acompanham o tema estudado.

O período de análise foi de 2010 a 2020, perfazendo um total de dez anos. Tal intervalo de tempo foi selecionado para se obter um panorama atualizado sobre as pesquisas, cujo tema seja qualidade da informação em cooperativas, podendo confrontar o panorama ora pesquisado, com os achados de pesquisas anteriores.

Para o levantamento da produção científica foram selecionados artigos de periódicos, congressos e dissertações que segundo Oliveira (2002) "representam uma relevante parte do fluxo de informação originado com a atividade científica de pesquisa”.

Quanto aos periódicos, foram selecionados aqueles que abordavam o tema: qualidade da informação e cooperativismo, tanto no panorama nacional, quanto no internacional, permitindo uma comparação entre os estudos desses dois cenários.

Por meio dos sites Scientific Periodicals Eletronic Library (SPELL) e Scholar Google, foram selecionados os artigos nacionais com os temas da pesquisa. Já em relação aos periódicos internacionais, foram consultados na base $W E B$ of Science, na área de pesquisa (SU) Business Economics.

O processo de seleção dos artigos nacionais ocorreu da seguinte forma: a) acessar o site SPELL; b) inserir os termos "Qualidade da Informação + Cooperativas", posteriormente, "Gerenciamento de Resultados + Cooperativas", "Escolhas Contábeis + Cooperativas", "Suavização + Cooperativas", "Conservadorismo + Cooperativas" e, por fim, "Relevância Contábil + Cooperativas" na ferramenta de busca do site; c) baixar os artigos que compreendiam ao período analisado, de 2010 a 2020.

Já o processo de seleção dos artigos internacionais se deu por meio da busca, nas bases já mencionadas, pelos termos: "Cooperative, Earnings Quality, Earnings management, Conservatism, Accounting Choices, Value Relevance, Credit Unions, Mutual company", que poderiam estar presentes no título, resumo e palavras-chave dos artigos, considerando o mesmo período de análise dos artigos nacionais.

Das buscas realizadas nos sites dos periódicos nacionais retornaram um total de 18 artigos, mas após análise dos conteúdos, apenas 14 artigos abordaram o tema. Já na procura por artigos internacionais, dentro dos parâmetros especificados anteriormente, retornaram 11 artigos pela base WEB of Science, mas após a exclusão dos artigos que não 
continham o assunto ligado à qualidade da informação realizada, de forma similar a dos trabalhos nacionais, restaram 3 artigos internacionais na amostra.

As informações extraídas pela leitura dos artigos, tais como, dados básicos do trabalho (título do artigo, nome dos autores, ano de publicação, nome do periódico e classificação pelo QUALIS/CAPES ou valor do JCR), a tipologia empregada (teórica ou empírica), a abordagem de pesquisa utilizada (qualitativa ou quantitativa), a metodologia de medição da qualidade da informação, o ramo de atividade das cooperativas analisadas e as palavras-chave, foram tabuladas com o auxílio do software Microsoft Excel.

Ainda com o auxílio dessa ferramenta, foram utilizadas técnicas de estatística descritiva nas análises de frequência dos dados, além da produção de gráficos que permitiram analisar padrões nas produções acadêmicas sobre qualidade da informação no cooperativismo e confrontá-las entre nacionais e internacionais.

Por fim, para corroborar e ilustrar a análise sobre os principais contextos em que a teoria da agência é utilizada, foi elaborada uma nuvem de palavras, a partir das palavras-chave, com o auxílio da ferramenta WordClouds.

\section{ANÁLISE E DISCUSSÃO DOS RESULTADOS}

Nesta seção foram apresentados os resultados após as análises dos artigos selecionados, tendo sido coletadas as informações de todos os artigos sendo possível conhecer os resultados apresentados na tabela 1.

Tabela 1 - Produção por periódico, congresso ou dissertação

\begin{tabular}{c|c}
\hline Periódico/Congresso & Quantidade \\
\hline Dissertação de Mestrado & 3 \\
Revista de Gestão e Organizações Cooperativas & 2 \\
Congresso USP - Controladoria e Contabilidade & 2 \\
Anpcont & 1 \\
ASAA & 1 \\
Brazilian Business Review & 1 \\
Congresso da Sober & 1 \\
International Review of Economics and Finance & 1 \\
Journal of Financial Services Research & 1 \\
Revista Ambiente Contábil & 1 \\
Revista Brasileira de Gestão de Negócios & 1 \\
The European Journal of Finance & 1 \\
USP International Conference in Accounting & 17 \\
\hline
\end{tabular}

Fonte: Dados da pesquisa (2020).

Na tabela 1 é possível verificar a dispersão das pesquisas com o tema de Qualidade da Informação em Cooperativas. Foram encontradas 3 dissertações que abordaram o tema. A Revista de Gestão e Organizações Cooperativas e o Congresso USP obtiveram 2 publicações cada e os demais artigos estão espalhados em outras revistas, com apenas 1 publicação sobre o tema no período analisado.

Na figura 1 podemos observar a evolução das publicações ao longo dos anos. Se verifica uma concentração de publicações nos anos de 2015 e 2018, com 4 publicações em cada um desses anos. Nos anos de 2010 e 2011 não houve publicações sobre o tema com amostra de cooperativas. 
Figura 1 - Evolução das publicações no tempo

\section{Quantidade}

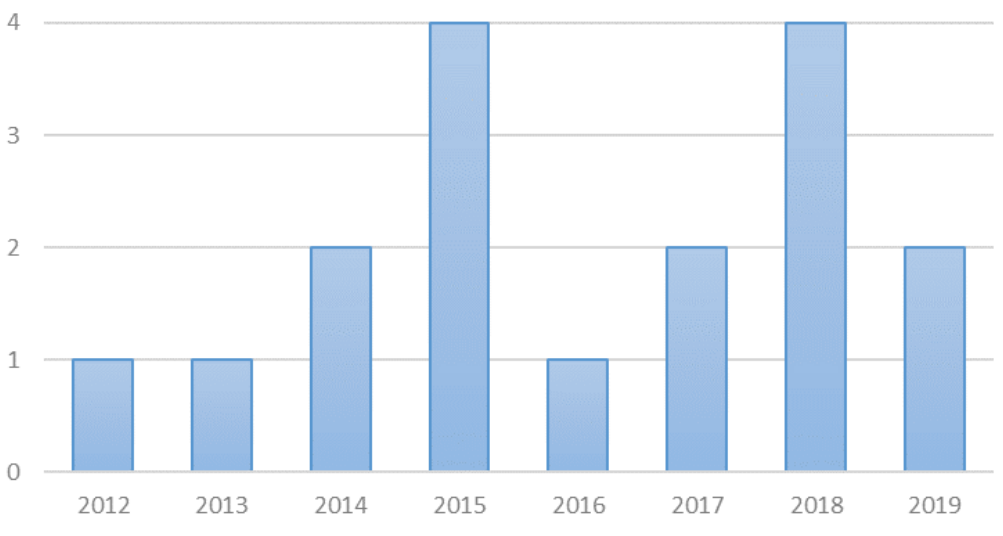

Na tabela 2, foram classificadas as produções científicas por autoria utilizando-se a Lei de Lotka. A lei determina que um grupo menor de pesquisadores produz a maior parte dos estudos, enquanto um grupo maior produz menos. Dessa forma, se consegue identificar quais os pesquisadores têm maior referência sobre o tema e quais têm maior produtividade (Machado Jr, Souza, Palmisano, Campanário \& Parisotto, 2014).

Tabela 2 - Lei de Lotka

\begin{tabular}{c|c|c}
\hline Artigos Publicados & Autores & Percentual do total de autores \\
\hline 6 & 1 & $3,45 \%$ \\
4 & 1 & $3,45 \%$ \\
3 & 1 & $3,45 \%$ \\
2 & 2 & $6,90 \%$ \\
1 & 24 & $82,76 \%$ \\
\hline
\end{tabular}

Fonte: Dados da pesquisa (2020).

Verifica-se na tabela 2 que apenas um autor possui seis publicações sobre o tema pesquisado, e que vinte e quatro autores somente participaram da publicação de um artigo na área. Apenas um autor possui 4 das publicações e outras 3 pertencem a um outro autor.

A tabela 3 evidencia os autores com maior produtividade no tema pesquisado. Para compô-la, foram selecionados apenas os autores com mais de uma publicação.

Tabela 3 - Principais autores sobre o tema

\begin{tabular}{c|c|c}
\hline Autor & & Quantidade \\
\hline Valéria Gama Fully Bressan & Universidade Federal de Minas Gerais & 6 \\
Aureliano Angel Bressan & Universidade Federal de Minas Gerais & 4 \\
José Marcos Da Silva Júnior & Universidade Federal de Minas Gerais & 3 \\
Lua Syrma Zaniah Santos & Universidade Federal de Minas Gerais & 2 \\
Saulo Cardoso Maia & Centro de Ensino Superior de Conselheiro Lafaiete & 2 \\
\hline
\end{tabular}

Fonte: Dados da pesquisa (2020).

V. Bressan e A. Bressan são os autores que mais produziram sobre qualidade da informação contábil em cooperativas, tendo produzido, respectivamente, seis e quatro trabalhos publicados. Posteriormente, são elencados os autores Da Silva Junior (três publicações), Santos e Maia (duas publicações). 
Dos cinco principais autores sobre o tema, quatro são vinculados à Universidade Federal de Minas Gerais (UFMG). Além disso, o autor Saulo Cardoso Maia, que é vinculado ao Centro de Ensino Superior de Conselheiro Lafaite, fez um de seus trabalhos como dissertação de mestrado justamente na UFMG.

Dessa forma, é possível confirmar a proposta estabelecida pela Lei de Lotka, visto que grande parte dos trabalhos são produzidos por um pequeno grupo de autores, tanto no cenário nacional quanto internacional.

Para se verificar a frequência das palavras-chave, foi elaborada a tabela 4 , na qual é possível verificar as palavras-chave mais citadas nas pesquisas da amostra.

Tabela 4 - Frequência das palavras-chave

\begin{tabular}{c|c}
\hline Palavra & Frequência \\
\hline Gerenciamento de resultados & $22,4 \%$ \\
Cooperativas de Crédito & $18,4 \%$ \\
Loan loss provisions & $6,1 \%$ \\
Cooperativas Agropecuárias & $4,1 \%$ \\
Income Smoothing & $4,1 \%$ \\
Sicredi & $4,1 \%$ \\
\hline
\end{tabular}

Fonte: Dados da pesquisa (2020).

Nota-se que as palavras-chave gerenciamento de resultados e cooperativas de crédito são as mais utilizadas nas pesquisas. Posteriormente, foram analisados quais temas e modelos de medição da qualidade da informação foram mais usados nas pesquisas.

Foi elaborada uma nuvem de palavras com base nas palavras-chave das pesquisas da amostra, conforme figura 2.

É possível verificar que as palavras em destaque são gerenciamento de resultados, cooperativas de crédito, cooperativas agrícolas, Loan Loos Provision (provisão para perdas) e Income Smoothing.

Figura 2 - Nuvem de palavras das palavras-chave

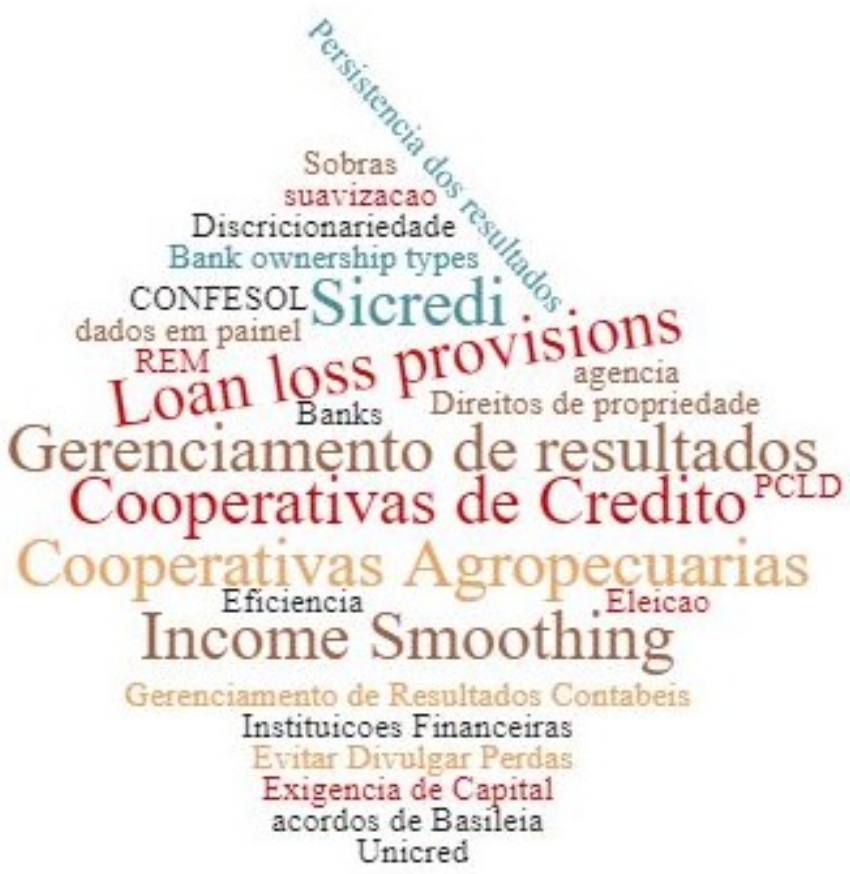

A tabela 5 foi construída para identificar qual método de qualidade da informação teria sido mais utilizado nas pesquisas com cooperativas. Sendo os resultados: gerenciamento de resultados (a suavização foi considerada separadamente), Value Relevance, conservadorismo e tempestividade. 
Tabela 5 - Metodologia de qualidade da informação contábil

\begin{tabular}{c|c}
\hline Método & Quantidade \\
\hline Gerenciamento de Resultados & 12 \\
Suavização & 4 \\
Persistência & 1 \\
\hline
\end{tabular}

Fonte: Dados da pesquisa (2020).

Analisando a tabela 5, é possível verificar que o gerenciamento de resultados é bastante explorado em pesquisas com cooperativas. Esse resultado encontrado pode ser utilizado para identificação de lacunas na literatura que possam ser preenchidas em pesquisas futuras. Adicionalmente, foi identificado o tema de persistência, que também é considerado na literatura como método de medição da qualidade da informação contábil.

Os resultados corroboram os estudos de Gomes et al. (2019) onde se percebeu que muitos estudos têm abordado aspectos ligados ao comportamento dos cooperados (gerenciamento de resultados) e de conflito de interesses.

Já os resultados apresentados na tabela 6, demonstram que o ramo de atividade das cooperativas mais explorado pelos autores que estudam qualidade da informação contábil foi o de cooperativas de crédito.

Tabela 6 - Ramo de atividade das cooperativas

\begin{tabular}{c|c}
\hline Ramo & Quantidade \\
\hline Crédito & 13 \\
Agropecuárias & 3 \\
Saúde & 1 \\
Total & 17 \\
\hline
\end{tabular}

Fonte: Dados da pesquisa (2020).

É possível, novamente, identificar lacunas nas pesquisas e verificar quais os tipos de cooperativas são menos explorados e suas particularidades. Segundo a OCB (2019), foram alterados os ramos de cooperativas para o ano de 2020 , passando de 13 para 8, são eles: agropecuário, crédito, consumo, infraestrutura, saúde, trabalho, produção de bens e serviços, transporte. Diante disso, pode-se notar que existem muitos ramos de atividade das cooperativas que são pouco explorados dentro do tema.

Na tabela 7 foram evidenciadas as análise dos modelos econométricos utilizados nas pesquisas. Assim, se pode estabelecer os modelos mais utilizados nesse tema e identificarmos novas oportunidades de pesquisas utilizando outros modelos presentes na literatura.

Tabela 7 - Modelos econométricos

\begin{tabular}{c|c}
\hline Autores & Quantidade \\
\hline Maia et al. (2013) & 4 \\
Burgstahler e Dichev (1997) & 3 \\
Dechow e Schrand (2004) & 2 \\
Ahmed et al. (1999) & 1 \\
Beatty e Liao (2011) & 1 \\
Bischoff e Lustosa (2014) & 1 \\
Greenawalt e Sinkey (1988) & 1 \\
Jones Modificado (1995) & 1 \\
Levantis et al. (2011) & 1 \\
Petroni (1992) & 1 \\
Roychowdhury (2006) & 1 \\
Total & 17 \\
\hline
\end{tabular}

Fonte: Dados da pesquisa (2020). 
Nota-se que não há uma predominância entre os modelos econométricos utilizados na base analisada, porém, destaca-se o modelo proposto por Maia et al. (2013), estando essa pesquisa, inclusive, contida na base analisada. O autor adequou o modelo de Ahmed et al. (1999) à realidade do cooperativismo brasileiro, utilizando-o em pesquisas com gerenciamento de resultados e suavização.

Dechow, Ge e Schrand (2010) qualificam as pesquisas em qualidade da informação contábil entre consequentes e determinantes. Pesquisas que testam consequências são aquelas nas quais a proxy de qualidade da informação é uma variável independente que busca analisar o impacto da qualidade da informação nos resultados. Entretanto, pesquisas com determinantes verificam se algumas variáveis da literatura poderiam explicar a qualidade da informação, sendo essa uma variável dependente.

Os resultados seguem a mesma linha de Gomes et al. (2019) na identificação dos modelos matemáticos utilizados nas pesquisas.

Desta forma, na tabela 8 se identifica os tipos de pesquisas, entre consequentes e determinantes.

Tabela 8 - Consequente/Determinante

\begin{tabular}{c|c}
\hline Tipo & Quantidade \\
\hline Consequente & 16 \\
Determinante & 1 \\
Total Geral & 17 \\
\hline
\end{tabular}

Fonte: Dados da pesquisa (2020).

Da tabela 8, verifica-se que no atual cenário das pesquisas, a qualidade da informação é medida como uma consequência das demais variáveis e não utilizada como uma variável independente.

\section{CONCLUSÃO}

O objetivo do presente trabalho foi verificar o panorama das pesquisas sobre qualidade da informação em um setor específico, que seria o de cooperativas. Utilizando-se das leis bibliométricas, foi possível observar descobertas relevantes.

Identificou-se os periódicos com maior volume de publicações com o tema abordado e a Revista de Gestão e Organização Cooperativas teve um destaque entre os periódicos, porém o maior número de pesquisas com esse tema é oriundo de dissertações.

Por meio da lei de Lotka foi verificado que $82,76 \%$ dos autores teriam publicado apenas um artigo com o tema da pesquisa. Constatou-se, ainda, que dois autores possuem um número relevante de publicações considerando o tamanho da amostra.

$\mathrm{Na}$ análise de frequência das palavras-chave foi possível identificar que os termos "gerenciamento de resultados" e "cooperativas de crédito" tiveram destaque. Esse resultado corroborou o resultado das tabelas 5 e 6 , que traziam o tipo de métrica de qualidade de informação e o ramo de atividade das cooperativas que seria mais explorado.

Uma possível explicação para a preferência pelas cooperativas de crédito se dá pela facilidade de extração de dados, que estão disponíveis no Banco Central. E por se tratar de uma cooperativa com atuação no setor financeiro, é natural que pesquisas em gerenciamento de resultados sejam mais exploradas, visto que é uma característica do setor.

Nas tabelas 7 e 8 foi constatado que existem diversos modelos utilizados para pesquisas em qualidade da informação, mesmo quando a maioria das pesquisas são de gerenciamento de resultados.

Outra informação importante obtida foi que a qualidade da informação é utilizada na maior parte dos trabalhos como uma variável dependente no modelo econométrico.

Como limitação do estudo ressalta-se a falta de pesquisas com o tema em tela. Mesmo considerando um período de dez anos, tendo sido necessária a inclusão de pesquisas apresentadas em congressos e dissertações ao presente estudo. Uma possível explicação para o baixo interesse de pesquisas nessa área é o fato de que poucas cooperativas fazem divulgação para o público, fazendo-a, em sua maioria, apenas para cooperados. É interessante ressaltar que mesmo no cenário internacional foram encontradas poucas publicações com o tema.

Para futuras pesquisas, sugere-se que sejam exploradas outras métricas de qualidade da informação, bem como outros ramos de atuação das cooperativas que possuem grandes particularidades.

\section{REFERÊNCIAS}

Ahmed, A. S. (1994). Accounting earnings and future economic rents: an empirical analysis. Journal of Accounting and Economics. 17, 377-400.

Almeida, J. E. F. D. (2010). Qualidade da informação contábil em ambientes competitivos (Doctoral dissertation, Universidade de São Paulo). 
Ball, R., Brown, P. (1968). An empirical evaluation of accounting income numbers. Journal of accounting research, 159-178.

Barth, M. E., Landsman, W. R., Lang, M. H. (2008). International accounting standards and accounting quality. Journal of accounting research, 46(3), 467-498.

Beaver, W. H. (1968). The information content of annual earnings announcements. Journal of accounting research, 67-92.

Beuren, I. M., \& Souza, J. C. (2007). Análise de Periódicos Internacionais de Contabilidade nas Dimensões da Qualidade "Finalidade do Produto" e "Mercado." In XXXI Encontro da ANPAD. Rio de Janeiro.

Blonkoski, P. R., Antonelli, R. A., \& Bortoluzzi, S. C. (2016). Contabilidade Gerencial: Análise Bibliométrica e Sistêmica da Literatura Cientifica Internacional. PRETEXTO, 18(1), 80-99.

Cook, M. L. (1995). The future of US agricultural cooperatives: A neo-institutional approach. American journal of agricultural economics, 77(5), 1153-1159.

Cook, M. L., Chaddad, F. R., \& Iliopoulos, C. (2004). Advances in cooperative theory since 1990: A review of agricultural economics literature. Erasmus University Rotterdam, Rotterdam School of Management.

Da Silva Vicente, S. C., Rafael, D. N., Serra, F. A. R., \& Almeida, L. R. T. (2020). Um estudo bibliométrico sobre CEOS nas estratégias empresariais. Revista Eletrônica de Estratégia \& Negócios, 13(2), 214-238.

Dechow, P., Ge, W., \& Schrand, C. (2010). Understanding earnings quality: A review of the proxies, their determinants, and their consequences. Journal of Accounting and economics, 50(2-3), 344-401.

Diniz, M. M. (2020). Qualidade da Informação Contábil: Um estudo das Cooperativas de Crédito Brasileiras (Dissertação (Mestrado). Programa de Pós-Graduação em Ciências Contábeis, Universidade Federal da Paraíba, João Pessoa, PB, Brasil. Recuperado em 11 maio, 2020 de http:// www.ccsa.ufpb.br/ppgcc/contents/documentos/dissertacoes/dissertacao-marcelo-maia-dinizdeposito.pdf/view.

Domingues, J. B. (2003). Uma contribuição à contabilidade das Sociedades Cooperativas: estudo de caso sobre a contabilização dos atos cooperativos e não cooperativos em uma cooperativa de produção vinícola do sul do Brasil, de acordo com a Norma Brasileira de Contabilidade-NBC T 10.8 (Doctoral dissertation).

Dunn, J. R. (1988). Basic cooperative principles and their relationship to selected practices. Journal of agricultural cooperation. $3(1), 83-93$

Fulton, M., \& Giannakas, K. (2001). Organizational commitment in a mixed oligopoly: Agricultural cooperatives and investor-owned firms. American journal of agricultural economics, 83(5), 1258-1265.

Gomes, A., Lima, S., Araújo, M., \& Asta, D. (2019). COOPERATIVAS AGRÍCOLAS: Uma Análise Bibliométrica da Produção. Revista de Gestão e Organizações Cooperativas, 33-52.

Hendriksen, E. S., Van Breda, M. F., \& Sokolov, J. V. (1997). Teoriya bukhgalterskogo ucheta [Accounting Theory]. Moscow, Finansy i statistika Publ, 576.

Imlau, J. M. (2015). Práticas de contabilidade gerencial em cooperativas de produção agropecuária do estado do Rio Grande do Sul.

Kothari, S. P., Ramanna, K., \& Skinner, D. J. (2009). What should GAAP look like? A survey and economic analysis.

Lopes, A. B., \& Martins, E. (2005). Teoria da contabilidade: uma nova abordagem.

LOPES, A. B. (2009). The relation between firm-specific corporate governance, cross-listing and the informativeness of accounting numbers in Brazil. 2009. Tese de Doutorado. The University of Manchester, Manchester.

Machado Jr.; C., Souza, M. D.; Palmisano, A.; Campanário, M.; Parisotto, I. D. S. (2014). Análise de viabilidade de utilizar as leis da bibliometria em diferentes bases de pesquisa. In: Associação Nacional De Pós-Graduação E Pesquisa Em Administração, XXXVIII EnANPAD. Anais... Rio de Janeiro: ANPAD.

Números do Cooperativismo Brasileiro. (2019). Acesso 11 de fevereiro de 2020, from: https://www.ocb.org.br/numeros

Oliveira, M. C. (2002). Análise dos Periódicos Brasileiros de Contabilidade. Revista Contabilidade \& Finanças - USP, 29, 68-86.

Österberg, P.; Nilsson, J. (2009). Members' perception of their participation in the governance of cooperatives: the key to trust and commitment in agricultural cooperatives. Agribusiness, 25 (2), 181-197.

Paulo, E. (2007). Manipulação das informações contábeis: uma análise teórica e empírica sobre os modelos operacionais de detecção de gerenciamento de resultados (Doctoral dissertation, Universidade de São Paulo).

Ribeiro Filho, J. F. (1993) Um Modelo de Análise de prestação de contas de Instituição Federal de Ensino Superior com vistas a melhor evidenciar a sua produtividade junto à sociedade - Um estudo de caso. Dissertação apresentada ao Departamento de Ciências Contábeis e Finanças do Rio de Janeiro - UERJ, 128.

Santos, L. S. Z., Bressan, V. G. F., Braga, M. J., \& Guerra, C. M. A. (2018). Gerenciamento de resultados e eficiência: um estudo nas cooperativas de crédito filiadas ao sistema Unicred. Revista de Gestão e Organizações Cooperativas, 5(10), 19-32.

Silva, S. S. D., Sousa, A. R. D., \& Leite, E. T. (2010). Conflito de agência em organizações cooperativas: um ensaio teórico. Organizações Rurais e Agroindustriais/Rural and Agroindustrial Organizations, 13(1511-2016-131377), 63-76.

Silva, R. L. M. (2013). Adoção completa das IFRS no Brasil: Qualidade das demonstrações contábeis e o custo de capital próprio (Tese de Doutorado em Ciências Contábeis, Departamento de Contabilidade e Atuária da Faculdade de Economia, Administração e Contabilidade, Universidade de São Paulo).

Staatz, J. M. (1987). Recent developments in the theory of agricultural cooperation. Journal of Agricultural Cooperation, 2(20), 74-95. 
Souza Junior, W. D. de, Costa, R. F.da S., Richetti, T. C. de S., Asta, D. D. (2018). O Impacto do gerenciamento de resultados por decisões operacionais no desempenho das cooperativas agropecuárias do Paraná. XII Congresso AnpCont.

Tarifa, M. R., \& de Almeida, L. B. (2019). A tipificação de cultura organizacional na perspectiva da contabilidade gerencial: um estudo em cooperativas agropecuárias. Enfoque: Reflexão Contábil, 38(1), 33-46.

Vitaliano, P. (1983). Cooperative enterprise: an alternative conceptual basis for analyzing a complex institution. American journal of agricultural economics, 65(5), 1078-1083.

Wang, D. (2006). Founding Family ownership and earnings quality. Journal of Accounting Research, 44(3).

Zylbersztajn, D.(1994). Organização de cooperativas: desafios e tendências. Revista de Administração, 29(3), 23-32. 